Universidade de São Paulo

Escola Superior de Agricultura "Luiz de Queiroz"

Dinâmica de incremento da área seccional do tronco e do dossel de povoamentos clonais de eucalipto em função de variáveis edafoclimáticas e nutricionais

Andréa Virginia Athayde Wenzel

Dissertação apresentada para obtenção do título de Mestra em Ciências, Programa: Recursos Florestais.

Opção em: Silvicultura e Manejo Florestal

Piracicaba

2019 


\section{Andréa Virginia Athayde Wenzel Engenheira Florestal}

Dinâmica de incremento da área seccional do tronco e do dossel de povoamentos clonais de eucalipto em função de variáveis edafoclimáticas e nutricionais versão revisada de acordo com a resolução CoPGr 6018 de 2011

Orientador:

Prof. Dr. JOSÉ LEONARDO DE MORAES GONÇALVES

Dissertação apresentada para obtenção do título de Mestra em Ciências, Programa: Recursos Florestais.

Opção em: Silvicultura e Manejo Florestal

\section{Piracicaba}


Dados Internacionais de Catalogação na Publicação DIVISÃO DE BIBLIOTECA - DIBD/ESALQ/USP

Wenzel, Andréa Virginia Athayde

Dinâmica de incremento da área seccional do tronco e do dossel de povoamentos clonais de eucalipto em função de variáveis edafoclimáticas e nutricionais / Andréa Virginia Athayde Wenzel. - - versão revisada de acordo com a resolução CoPGr 6018 de 2011. - - Piracicaba, 2019.

$52 \mathrm{p}$.

Dissertação (Mestrado) - - USP / Escola Superior de Agricultura "Luiz de Queiroz".

1. Fertilização mineral 2. Índice de área foliar 3. Dendrômetro 4. Sazonalidade climática I. Título 


\section{DEDICATÓRIA}

À minha mãe, Maria Aparecida, pelo exemplo de garra e determinação.

Aos meus tios, Maria Teresinha e Narcy, por todo o apoio e incentivo, principalmente aos estudos.

Aos meus irmãos, Natalia e Alexandre.

Dedico

A minha avó Isolina (in memoriam), pelos cuidados e ensinamentos transmitidos.

Ofereço 


\section{AGRADECIMENTOS}

À Deus pelo dom da vida, saúde e sabedoria.

À Nossa Senhora de Schoenstatt, por se fazer presente em minha vida e colocar em meu caminho pessoas incríveis, que contribuiram significativamente para a conclusão deste trabalho e o meu crescimento pessoal;

Ao Prof. José Leonardo de Moraes Gonçalves, por esta oportunidade e demais confiadas, aos ensinamentos e compreensão para com os períodos difíceis de minha vida e decisões tomadas.

À Escola Superior de Agricultura "Luiz de Queiroz", à Universidade de São Paulo e ao Programa de Pós Graduação em Recursos Florestais, bem como, a todos os funcionários que contribuiram para minha formação profissional desde a Graduação;

Ao Programa Cooperativo sobre Silvicultura e Manejo (PTSM) do Instituto de Pesquisa e Estudos Florestais - IPEF, pelo apoio logístico e financeiro; e ao José Carlos Arthur Júnior por todo o apoio à este trabalho;

À FAPESP pela bolsa de Iniciação Científica concedida (Processo: 2013/24919-3);

À Capes pela bolsa de Mestrado concedida (Código: 33002037021M-8);

Aos meus grandes amigos Eduardo A. S. C. Melo, José Henrique T. Rocha, Carolina B. Brandani e Estela Foltran, pela confiança, apoio nas atividades e nas discussões deste trabalho. Sou grata a cada ensinamento transmitido e oportunidade de aprendizado.

Aos estagiários do PTSM, em especial a Amanda F. Franci, Ana R. S. Zucon e Felipe B. Saldanha, por todo o apoio nas atividades de campo. Vocês foram imprescindíveis para que eu conseguisse concretizar este trabalho em minha Graduação. Sou grata pela amizade, dedicação e a todos os momentos que divimos - aos bons e aos de dificuldade que superamos juntos.

Às Empresas Florestais participantes da Rede Experimental Aduba/PTSM e aos engenheiros que possibilitaram a realização deste trabalho: Ramires Reflortec Ltda - Rogérios R. Malheiros, Duratex S/A - Jarbas S. Borges, e International Paper do Brasil - Cristiane C. Z. de Lemos e Rodrigo E. Hakamada;

Aos Professores Paulo C. Sentelhas, Mario Tomazello Filho, Ricardo F. de Oliveira, Fábio R. Marin e Júlio C. Neves, pela oportunidade de discussão e palavras de incentivo;

Aos Pesquisadores Deon Malherbe, Sven-Olof Lundqvist, Andrew Carpenter, Rosana C. V. Higa, Mauricio M. Motter e Clayton A. Alvares, pelo reconhecimento do trabalho proposto, oportunidade de discussão e incentivo à continuidade dos estudos acadêmicos; 
EPÍGRAFE

A esperança, como a fênix, pode voar pelos céus do deserto e, ainda, desafiando o despeito do destino, reviver das cinzas e crescer.

Miguel de Cervantes Saavedra 


\section{RESUMO}

\section{Dinâmica de incremento da área seccional do tronco e do dossel de povoamentos clonais de eucalipto em função de variáveis edafoclimáticas e nutricionais}

As plantações comerciais de Eucalyptus no Brasil destacam-se mundialmente, pela produtividade alcançada no manejo de curta rotação. Os elevados índices obtidos são o reflexo da adaptabilidade às condições edafoclimáticas, do melhoramento genético e de práticas silviculturais adotadas. Entretanto, o desenvolvimento dessas plantações sofre influência de agentes bióticos e abióticos, sendo os de maior limitação nos trópicos a disponibilidade hídrica e a fertilidade do solo. O objetivo com esta dissertação foi, portanto, avaliar o efeito da fertilização completa com NPK e de omissões isoladas de cada nutriente, no crescimento de povoamentos clonais (entre 48 e 60 meses de idade) e, respostas as variações climáticas no ritmo de incremento do tronco e no índice de área foliar (IAF). Este estudo foi conduzido em 4 sítios florestais, localizados nos Estados de São Paulo e Mato Grosso do Sul, diferindo quanto ao material genético (híbridos E. grandis $x$ urophylla e E. urophylla $x$ globulus), tipos climáticos (Cwa e Aw) e classes de solo (Latossolo Vermelho, Latossolo Vermelho-amarelo e Neossolo quartzarênico). O delineamento experimental consistiu de cinco tratamentos e quatro repetições. Faixas dendrométricas permanentes foram utilizadas para monitorar o incremento do tronco das árvores, a cada 15 dias, ao longo de 12 meses. De cada tratamento, 15 árvores foram selecionadas, de acordo com a circunferência a altura do peito e desvios. O IAF foi estimado pelo método indireto de fotografias hemisféricas, mensalmente, por meio de 16 imagens do dossel de cada tratamento. Nos sítios, a produtividade de madeira variou entre 52 e $22 \mathrm{~m}^{3} \mathrm{ha}^{-1}$ $\mathrm{ano}^{-1}$, sendo limitante as omissões isoladas de $\mathrm{N}$ e de K. Condições atípicas do clima de 2014 afetaram significativamente o crescimento dos povoamentos, principalmente em São Paulo, no qual a redução da precipitação pluvial anual variou entre 35 e $74 \%$ (em relação a normal climatológica - 2008/20014). O incremento médio acumulado em área da secção transversal do tronco (ASTt) variou de $8,6 \mathrm{~cm}^{2}$ a $13,4 \mathrm{~cm}^{2}$. As oscilações entre máximo e mínimo incremento da ASTt, diferiram entre o grau de estresse e heterogeneidade dos povoamentos (maiores e menores árvores), o que pode ser explicado em até $73 \%$ pelas variáveis precipitação pluvial, amplitude térmica e temperatura média. Os tratamentos mais afetados pelo clima foram os de fertilização completa e omissão de K. Não houve diferença do IAF entre tratamentos, apenas em relação a sazonalidade climática.

Palavras-chave: Dendrômetro; Fertilização mineral; Sazonalidade climática; Mudança climática. 


\section{ABSTRACT \\ Dynamics of increment of the sectional area of the trunk and the canopy of clonal eucalypt stands as a function of edaphoclimatic and nutritional variables}

Commercial plantations of Eucalyptus in Brazil stand out worldwide, for the high productivity in the managements of short rotation. The high indexes obtained are the reflection of adaptability to the edaphoclimatic conditions, the genetic improvement and the silvicultural practices adopted. However, the development of these plantations is influenced by biotic and abiotic agents, which in the tropics the most limiting factors are water availability and soil fertility. The objective of this dissertation was, therefore, to evaluate the effect of complete NPK fertilizationand the isolated omissions of each nutrient on the growth of clonal stands (between 48 and 60 months of age) and, responses to climatic variations in the dynamic of increment of the trunk and leaf area index (LAI). This study was conducted in four forest sites, located in the states of São Paulo and Mato Grosso do Sul, differing in genetic material (hybrids E. grandis $x$ urophylla and E. urophylla $x$ globulus), climatic types (Cwa and Aw) and soil classes (Quartzipsammen, Rhodic Hapludox, Typic Hapludox). The experimental design consisted of five treatments and four replicates. Permanent dendrometric bands were used to monitor the increment of the tree's stems, measuring it each 15 days, for 12 months. From each treatment, 15 trees were selected, according to the circumference of the mean chest height and deviations. The LAI was estimated by the indirect method of hemispheric photographs, monthly, using 16 images of the canopy of each treatment. At the sites, wood productivity varied between 52 and $22 \mathrm{~m}^{3} \mathrm{ha}^{-1}$ year ${ }^{-1}$, being limiting the isolated omissions of $\mathrm{N}$ and $\mathrm{K}$. Atypical conditions of the climate of 2014 significantly affected the growth of the stands, mainly in São Paulo, in which the annual rainfall reduction varied between 35 and $74 \%$ (in relation to the normal climate - 2008/2014). The mean cumulative increase in trunk crosssectional area (TCSA) ranged from $8,6 \mathrm{~cm}^{2}$ to $13,4 \mathrm{~cm}^{2}$. The variations between maximum and minimum increment of TCSA differed between the degree of stress and heterogeneity of stands (larger and smaller trees), which can be explained in up to $73 \%$ by the variables rainfall, thermal amplitude and average temperature. The treatments most affected by the climate were those of complete fertilization and omission of $\mathrm{K}$. There was no difference in LAI between treatments, only in relation to climatic seasonality.

Keywords: Dendrometer; Mineral fertilization; Climatic seasonality; Climate chance. 


\title{
1. DINÂMICA DE INCREMENTO DA ÁREA SECCIONAL DO TRONCO E DO DOSSEL DE POVOAMENTOS CLONAIS DE EUCALIPTO EM FUNÇÃO DE VARIÁVEIS EDAFOCLIMÁTICAS E NUTRICIONAIS
}

\section{RESUMO}

A produtividade de plantações de eucalipto está vinculada às características do sítio, do material genético e das práticas silviculturais adotadas, em sua implantação e condução. A fertilidade do solo, assim como, a precipitação pluvial e temperatura ambiente são os agentes abióticos de maior limitação ao desenvolvimento das árvores, com reflexos no ritmo de incremento diamétrico do tronco e índice de área foliar (IAF). Neste contexto, este estudo teve como objetivos avaliar o efeito da fertilização mineral completa com NPK e omissões isoladas de cada nutriente no crescimento das árvores, bem como, as respostas do povoamento florestal às condições climáticas. $\mathrm{O}$ estudo foi conduzido em quatro locais, diferindo quanto ás condições edafoclimáticas e material genético (híbridos E. grandis $x$ urophylla e E. urophylla $x$ globulus). O delineamento experimental consistiu de blocos casualizados, com cinco tratamentos e quatro repetições. Faixas dendrométricas permanentes foram utilizadas para monitorar o incremento do tronco das árvores a cada 15 dias, ao longo de 12 meses. De cada tratamento, quinze árvores foram selecionadas e subdivididas em cinco classes diamétricas, de acordo com a circunferência a altura do peito média e desvios de 0,5 até 2,5. O índice de área foliar foi determinado pelo método indireto de fotografias hemisféricas, mensalmente, por meio de quatro imagens dossel de cada parcela. Condições atípicas do clima, como a redução da precipitação pluvial entre 35 e 74\%, afetaram significativamente a dinâmica de crescimento árvores, sendo os tratamentos de menor incremento do tronco o NPK e omissão de K. Oscilações entre máximo e mínimo crescimento foram observadas, diferindo quanto grau de estresse hídrico e heterogeneidade do povoamento (maiores e menores árvores). A precipitação pluvial, a temperatura média e amplitude térmica local foram as variáveis climáticas de maior influência no crescimento das árvores.

Palavras-chave: Dendrômetro; Fertilização mineral; Sazonalidade climática; Mudança climática.

\begin{abstract}
The productivity of eucalyptus plantations is linked to the characteristics of the site, the genetic material and the silvicultural practices adopted, in its implementation and conduction. Soil fertility, as well as rainfall and ambient temperature are the most limiting abiotic agents to the development of trees, with reflexes in the rhythm of diameter increase of the trunk and leaf area index (LAI). In this context, the objective of this study was to evaluate the effect of complete mineral fertilization with NPK and isolated omissions of each nutrient on tree growth, as well as the responses of forest stands to climatic condicions. The study was conducted at four sites, differing in soil and climatic conditions and genetic material (hybrids E. grandis $x$ urophylla and E. urophylla $x$ globulus). The experimental design consisted of randomized blocks, with five treatments and four replications. Permanent dendrometric bands were used to monitor the increment of the trunk of the trees every 15 days, during 12 months. From each treatment, fifteen trees were selected and subdivided into five diametric classes, according to the circumference of the mean chest height and deviations of 0,5 to 2,5 . The leaf area index was determined by the indirect method of hemispheric photographs, monthly, through four canopy images of each plot. Atypical climatic conditions, such as the reduction of rainfall between 35 and $74 \%$, significantly affected the growth dynamics of trees, being the traits of smallert increment of the trunk the NPK and omission of K. Oscillations between maximum and minimum growth were observed, differing the degree of water stress and heterogeneity of the stands (higher and smaller trees). Rainfall, mean temperature and local thermal amplitude were the climatic variables with the greatest influence on tree growth
\end{abstract}

Keywords: Dendrometer; Mineral fertilization; Climatic seasonality; Climate chance. 


\subsection{Introdução}

No Brasil, o gênero Eucalyptus é o mais empregado em plantações comerciais $(5,7$ milhões de hectares) sendo, em sua maioria, estabelecidas em solos de baixa aptidão agrícola e reservas minerais, com predomínio de caulinita, óxidos e hidróxidos de ferro e alumínio (Barro \& Novais, 1996; Brandani et al., 2017, Ibá, 2017). Neste cenário, a produtividade de

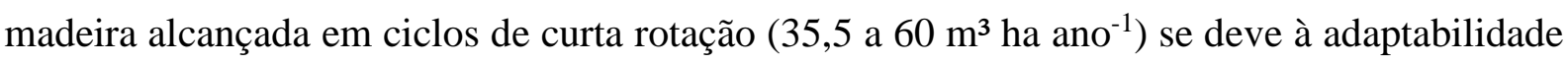
dos genótipos às condições edafoclimáticas, à capacidade de absorção e uso de nutrientes, à seleção e melhoramento genético, além do manejo silvicultural apropriado, com a gestão e aplicação de fertilizantes minerais, espaçamento de plantio e controle de plantas invasoras (Powers, 1999; Santana et al., 2002; Gonçalves et al., 2004; Stape et al; 2010; Gonçalves et al.; 2013; Gonçalves et al., 2017; Ibá, 2017,2018).

Em plantações clonais de Eucalyptus, a demanda nutricional por N, P, K, Ca e Mg é elevada, tornando a fertilização mineral e demais práticas conservacionistas, como o cultivo mínimo do solo, essenciais (Gonçalves et al., 2008; Rocha et al., 2016). Embora respostas sejam mais recorrentes para P e K nos trópicos e subtrópicos (Laclau et al., 2010; Gonçalves et al., 2011), aplica-se doses de N, Ca e Mg, como fertilização de reposição (Pulito et al., 2015; Melo et al., 2016). As respostas à fertilização mineral em volume de madeira são variáveis, entre 30 e 50\%, em função do nutriente, idade de avaliação, demanda nutricional do genótipo escolhido e condições edafoclimáticas (Downes et al., 1999, Laclau et al., 2009, 2010; Smethurst, 2010; Du Toit et al., 2010; Battie-Laclau et al., 2014; Bazani, 2014).

O incremento volumétrico das plantações sofre influência das condições climáticas, pois afetam a atividade do meristema cambial e, a relação funcional existente entre o dossel e área transversal do alburno das árvores (Kriedemann \& Cromer, 1996; Macedo et al, 2014; Nikinmaa et al., 2014; Sette Jr. et al., 2017; Oladi et al., 2017). Após o fechamento do dossel, em que o índice de área foliar é máximo, a competição intraespecífica entre árvores por recursos do meio aumenta (Du Toit et al., 2008; Gonçalves, 2011), sendo a água o fator abiótico de maior limitação a produtividade nos trópicos (Stape et al, 2010; Binkley et al., 2017; Christina et al., 2018), com reflexos significativos nas taxas de incremento do tronco e qualidade da madeira (Laclau et al., 2005; Drew et al, 2009; Sette Jr. et al, 2016; Castro et al., 2017).

A influência climática no incremento volumétrico das árvores tem por elementos: i. a radiação solar, que atua como fonte de energia para excitação da clorofila (faixa do espectro visível - 0,40 a $0,70 \mu \mathrm{m})$ e produção de carboidratos, por meio da fotossíntese; ii. a 
temperatura ambiente, que influência processos fisiológicos como fotossíntese, respiração, transpiração, divisão celular e fases fenológicas; e iii. a disponibilidade hídrica do solo, que regula a abertura estomática, devido aos processos de balanço de água interno e perda por transpiração (Souza et al., 2006; Rody et al., 2014).

A mediação continua, em períodos curtos de tempo, do diâmetro do tronco e do dossel das árvores possibilita avaliar os efeitos das condições climáticas e da fertilização mineral (Bréda et al., 2003; Laclau et al., 2007; Sette Jr. et al., 2012; Swaef et al., 2016, Bussotti et al., 2017). Ferramentas como o dendrômetro permanente tem-se mostrado uma opção eficiente e de baixo custo, para avaliação do incremento do tronco das árvores (Botosso \& Tomazello Filho, 2001; Sette Jr. et al., 2012; Shimamoto et al., 2015). Quanto ao dossel, de forma não destrutiva, pode ser avaliado por meio de fotografias hemisféricas, que possibilitam a estimativa do índice de área foliar (Xavier et al., 2002; Jonckheere et al., 2004; Guinti Neto et al., 2015).

Sabendo-se da influência que a fertilização mineral desempenha na produtividade florestal e da influência das condições climáticas na dinâmica de incremento do diâmetro do tronco, propôs-se as seguintes hipóteses:

I. A resposta em incremento da área da secção transversal do tronco das árvores em povoamentos fertilizados tem, primariamente, estreita relação com a disponibilidade hídrica e térmica;

II. A fertilização mineral com N, P e K melhora a aclimatação do material genético, diminuindo as oscilações na dinâmica de incremento do tronco e área foliar de plantações clonais de eucalipto;

III. A adaptabilidade do material genético as condições edafoclimáticas promove maior homogeneidade entre árvores e menor oscilação da dinâmica de crescimento em períodos de restrição hidríca;

Objetivou-se com este trabalho, avaliar ao longo de 12 meses: i. os padrões de crescimento de plantações clonais estabelecidas sob omissão da fertilização com N, P e K em quatro condições edafoclimáticas distintas, e idades entre 48 e 60 meses; ii. avaliar a influência das condições edafoclimáticas na dinâmica de crescimento das plantações; iii. avaliar a relação existente entre índice de área foliar (IAF) e sazonalidade climática; 


\subsection{Conclusão}

O incremento em área da secção tranversal do tronco das árvores (ASTt) foi afetado pelas variáveis climáticas e tratamentos de fertilização mineral. Todavia, a disponibilidade hídrica foi o fator mais limitantes em 2014, principalmente para os sítios do Estado de São Paulo, devido à redução da precipitação pluvial anual.

O comportamento em crescimento das árvores, quanto ao ganho e redução da área ASTt, pode ser explicado pela precipitação pluvial, temperatura média e amplitude térmica. As diferenças observadas, ocorreram em função da adaptabilidade dos materiais genéticos às condições do meio, heterogeneidade do povoamento e grau de estresse.

Não houve diferença na dinâmica de incremento da ASTt entre tratamentos. Entretanto, diferenças foram observadas no incremento acumulado total, sendo menor para os tratamentos de fertilização completa e omissão isolada de K. As respostas se relacionam a redução hídrica abrupta e a alta taxa metabólica promovida pelo NPK, bem como, aos efeitos da deficiência de $\mathrm{K}$.

As oscilções negativas da ASTt (contração), provavelmente, ocorreram pela redução do teor de água das células da casca interna (tecidos elásticos de armazenamento), para a manutenção do balanço hídrico das árvores, devido a transpiração e déficit de água no solo.

Não houve diferença do IAF entre tratamentos, apenas em relação a sazonalidade climática.

\section{Referências}

Adams, H. D.; Zeppel, M. J. B.; Anderegg, W. R. L.; Hartmann, H.; Landhäusser, S. M.; Tissue, D. T. et al.; 2017. A multi-species synthesis of physiological mechanisms in drought-induced tree mortality. Nature Ecology and Evolution, 1, 1285-1291.

Allen, R.G., Pereira, L.S., Raes, D. e Smith, M. 1998. Crop Evapotranspiration Guidelines for

Compu ting Crop Water Requirements. Rome, Food and Agriculture Organization of the United Nations. (FAO Irrigation and Drainage, Paper 56).

Almeida, A. C.; Soares, J. V.; Landsberg, J. J., Rezende,G. D. 2010. Growth and water balance of Eucalyptus grandis hybrid plantations in Brazil during a rotation for pulp production. Forest Ecology and Management, 251, 10-21. 
Aspinwall, M. J., King, J. S., Mckeand, S. E., Bullock, B. P. Genetic effects on stand-level uniformity and above and belowground dry mass production in juvenile loblolly pine. Forest Ecology and Management, 262, 609-619.

Alcarde, C. A., Stape, J. L., Sentelhas, P. C., Gonçalves, J. L. M., Sparovek. 2013. Koppens's climate classification map for Brazil. Meteorologische Zeitschrift, 6, 711-728.

Barros, N. F.; Comerford, N. B. Sustentabilidade da produção de florestas plantadas na região tropical. 2002. In: Alvarez V., V. H.; Schaefer, C. E. G. R.; Barros, N. F.; Melo, J. W. V.; Costa, L. M.. Tópicos em ciência do solo. Viçosa/Soc. Bras. De Ciência do Solo, pp. 487-592.

Barros, N. F.; Novais, R. F. 1996. Eucalipt nutrition and fertilizer regimes in Brasil. In: Attiwill, P. M.; Adams, M. A. Nutrition of Eucalypts. Australia/CSIRIO, pp. 335-356.

Battaglia, M.; Cherry, M.L.; Beadle, C.L.; Sands, P.J.; Hingston, A. 1998. Prediction of leaf area index in eucalypt plantations: Effects of water stress and temperature. Tree Physiol., 18, 521-528

Battie-Laclau, P.; Laclau, J. P.; Domec, J. C.; Christina, M.; Bouillet, J. P.; Piccolo, M. C.; Gonçalves, J. L. M.; Moreira, R. M.; Krusche, A. V.; Bouvet, J. M.; Nouvellon, Y. 2014. Effects of potassium and sodium supply on drought adaptive mechanisms in Eucalyptus grandis plantation. New Phytologist, 203, 401-413.

Battie-Laclau, P., Laclau, J. P., Picoolo, M. C. 2013. Influence os potassium and sodium nutrition on leaf area components in Eucalyptus grandes trees. Plant of Soil, 371, 19-35.

Bazani, J. H. 2014. Eficiência de fertilizantes fosfatados solúveis e pouco solúveis, com ou sem complexação com substâncias húmicas, em plantações de eucalipto. In: Ciências Florestais. USP, Piracicaba - SP, pp. 129

Binkley, D., Campoe, O. C., Gspaltl, M., Forrester, D. J. 2013. Light absorption and use efficiency in florests: Why patterns differ for trees and stands. Forest Ecology and Management, 288, 5-13.

Binkley, D., Campoe, O. C., Alvares, C., Carneiro, R. L., Cegatta, I., Stape, J. L. 2017. The interations of climate, spacing, and genetics on clonal Eucalyptus plantations across Brazil and Uruguay. Forest Ecology and Management, 405, 271-283.

Binkley, D. 2004. A hypothesis about the interaction of tree dominance and stand production through stand development. Forest Ecology and Management, 190, 265-271.

Botosso, P. C; Tomazello Filho, M. 2001. Aplicação de faixas dendrométricas na dendrologia: avaliação da taxa e do ritmo de crescimento do tronco das árvores tropicais e subtropicais. In: Maia, N. B.; Martos, H. L., Barrella, W. Indicadores ambientais: conceitos e aplicações. São Paulo: EDUC, 7,147-171.

Brandani, C.B.; Rocha, J.H.T.; Godinho, T. O.; Wenzel, A.V.A.; Gonçalves, J.L.M. 2017. Soil C and $\mathrm{Al}$ availability in tropical single and mixed-species of Eucalyptus sp. and Acacia mangium plantations. Geoderma Regional, 10, p. 85-92.

Bréda, N. J. J. 2003. Ground-based measurements of leaf area index: a review of methods, instruments and current controversies. Journal of Experimental Botany, 54, 2043-2417. 
Biondi, F., Rossi, S. 2015. Plant-water relationships in the Great Basin Desert of North America derived from Pinus monophylla hourly dendrometer records. Int J Biometeorol, 59, 939-953.

Bovard, B. D.; Curtis, P. S.; Vogel, C. S.; Su, H. B.; Schmid, H. P. 2005. Environmental controls on sap flow in a northern hardwood forest. Tree Physiology, 25, 31-38.

Bussotti, F.; Pollastrini, M. 2017. Traditional and novel indicators of climate change impacts on European Forest trees. Forests, 8. 1-12.

Campoe, O. C.; Munhoz, J. S. B.; Alvares, C. A.; Carneiro, R. L.; Mattos, E. M.; Ferez, A. P. C.; Stape, J. L. 2016. Meteorological seasonality affecting individual tree growth in forest plantations in Brazil. Forest Ecology and Management, 380, p. 149-160.

Campoe, O. C. Stape, J. L.; Nouvellon, Y.; Laclau, J. P.; Bauerle, W. L.; Binkley, D.; Le Maire, G. 2013. Stem production, light absorption and light use efficicency between dominant and nondominant trees of Eucalyptus grandis across a productivity gradient in Brazil. Forest Ecology and Management, 288, 14-20.

Castro, V. R.; Surdi, P. G.; Filho, M. T.; Chaix, G.; Laclau, J. P. 2017. Efeito da disponibilidade hídrica e da aplicação de potássio e sódio no crescimento em diâmetro do tronco das árvores de Eucalyptus grandis. Scientia Forestalis, 45, 89-99.

Christina, M., Le Maire, G., Nouvellon, Y., Vezy, R., Bordon, B., Battie-Laclau, P., Gonçalves, J. L. M., Delgado-Rojas, J. S., Bouillet, J. P., Laclau, J. P. 2018. Simulation the effects of different potassium and water supply regimes on soil water content and water table depth over rotation of a tropical Eucalyptus grandis plantation. Forest Ecology Management, 418, 4-14.

Christina, M., Nouvellon, Y., Laclau, J. P., Stape, J. L., Bouillet, J. P., Lambais, G. R., Le Maire, G. Importance of deep water uptake in tropical eucalypt forest. Functional Ecology, 31, 509-519.

Downes, G.; Beadle, C.; Worledge, D. 1999. Daily stem growth patterns in irrigated Eucalyptus globulus and E. nitens in relation to climate. Trees,14, 102-111.

Drew, D. M., O‘ Grady, A. P., Downes, G. M., Read, J., Worledge, D. 2008. Daily patterns of stem size variation in irrigated and non-irrigated Eucalyptus globulus. Tree Physiology, 28, 1573-1581.

Drewn, D. M.; Downs, G. M.; Grzeskowiak, V.; Naiodoo, T. 2009. Differences in daily stem sixe variation and growth in two hybrid eucalypt clones. Trees, 23, 585-595.

Du Toit, B. 2008. Effects of site management on growth, biomass partitioning and light use efficiency in a young stand of Eucalyptus grandis in South Africa. Forest Ecology and Management, 255, 2324-2336.

Du Toit, B; Smith, C. W., Little, K. M., Boreham, G., Pallet, R. N. 2010. Intensive, site-specific silviculture: Manipulating recorse availability at establishment for improved stand productivity. A review of South African research. Forest Ecology and Management, 259, 1836-1845.

Ferreira, J. M. A; Stape, J. L. 2009. Productivity gains by fertilisation in Eucalyptus urophylla clonal plantations across gradients in site and stand conditions. Souther Forests, 71, 253-258.

Fischer, R. F; Binkley, D. 2000. Ecology and management of forest soils. John Wiley \& Sons, Inc. 
Forrester, D. I.; Medhurst, J. I.; Wood, M.; Beadle, C. L.; Valencia, J. C. 2010. Growth and physiological responses to silviculture for producing solid-wood products from Eucalyptus plantations: An Australian perspective. Forest Ecology and Management, 259, 1819-1835.

Gava, J. L, Gonçalves, J. L. M. 2008. Soil attributes and wood quality for pulp production in plantations of Eucalyptus grandis clone. Sci. Agric., 65, 306-313.

Gentil Otto, M. S., Hubbard, R. M., Binkley, D., Stape, J. L. 2014. Dominant clonal Eucalyptus grandis x urophylla trees use water more efficiently. Forest Ecology and Management, 328, 117 121.

Gonçalves, J. L. M.; Alvares, C. A.; Rocha, J. H. T.; Brandani, C. B.; Hakamada, R. E. 2017. Eucalypt plantation management in regions with water stress. Southern Forests, 79, 85-92.

Gonçalves, J. L. M.; Alvares, C. A.; Higa, A. R.; Silva, L. D.; Alfenas, A. C.; Stahl, J.; Ferraz, S. F. B.; Lima, W. P.; Brancalion, P. H. S.; Hubner, A.; Bouillet, J. P. L.; Nouvellon, D. E. 2013. Integrating genetic and silvicultural strategies to minimize abiotic and biotic constraints in Brasilian eucalypt plantations. Forest Ecology and Management, 301, 6-27.

Gonçalves, J. L. M. 2011. Fertilização de Plantações de Eucalipto. In: Gonçalves, J. L. M. G.; Pulito, A. P.; Arthur Junior, J. C.; Silva, L. D. In: Encontro Brasileiro de Silvicultura, 2., Anais... Piracicaba/IPEF, pp. 85-109.

Gonçalves, J. L. M.; Stape, J. L.; Laclau, J. P.; Bouillet, J. P.; Ranger, J. 2008. Assessing the effects of early silvicultural management on long-term site productivity of fast-growing eucalypt plantations: the Brazilian experience. Southern Forests, 70, 105-118.

Gonçalves, J. L. M.; Stape, J. L.; Laclau, J. P.; Smerthurst, P.; Gava, J. L.; 2004. Silvicultural effects on the productivity and wood quality of eucalypt plantations. Forest Ecology and Management,193, 45-61.

Hakamada, R. E., Stape, J. L., Lemos, C. C. Z., Almeida, A. E. A., Silva, L. F. 2015a. Uso do inventário florestal e da uniformidade entre árvores como ferramenta de monitoramento da qualidade silvicultural em plantios de eucalipto. Scientia Forestalis, 43, 27-36.

Hakamada, R. E., Stape, J. L., Lemos, C. C. Z., Almeida, A. E. A., Silva, L. F. 2015b. Uniformidade entre árvores durante uma rotação e sua relação com a produtividade em Eucalyptus clonais. Cerne, 21, 465-472.

Hawkesford, M., Horst. W., Kichey, T., Lambers, H., Schjoerring, J., Skrumsager, M., White, P. Functions of Macronnutrients. 2012. In: Marschner, P. Marschner's Mineral Nutrition of higher plants. Elsevier, pp. 135-189.

Hodecker, B., E. R.; Barbosa, A. P., Barros, N. F.; Merchant, A. 2018. Water availability preceding long-term drought defines the tolerance of Eucalyptus to water restriction. New Forests, 49, 173 195.

Hölttä, T.; Harri, M.; Nojd, P.; Makela, A.; Nikinmaa, E. A physiological model of softwood cambial growth. Tree Physiology, 30, 1235-1252. 
Hölttä, T.; Vesala, T.; Sevanto, S.; Perämäki, M.; Nikinmaa, E. 2006. Modeling xylem and phloem water flows in trees according to cohesing theory and Münch hypothesis. Trees, 20, 67-78.

IBÁ 2017. Anuário estatístico da Industria Brasileira de Árvores - ano base 2016, São Paulo.

IBÁ 2018. Sumário estatístico da Indústria Brasileira de Árvores - ano base 2017. São Paulo.

Jesus, G. L.; Barros, N. F.; Silva, I. R.; Neves, J. C. L.; Henriques, E. P., Lima, V. C.; Fernandes, L. V.; Soares, E. M. B. 2012. Doses e fontes de nitrogênio na produtividade do eucalipto e nas frações da matéria orgânica em solo da região do cerrado de Minas Gerais. R. Bras. Ci. Solo, 36, 201-214.

Jonckheere, I., Fleck, S., Nackaerts, K., Muys, B., Coppin, P., Weiss, M., Baret, F. 2004. Review of methods for in situ leaf area index determination Part I. Theories, sensors and hemispherical photography. Agricultural and Forest Meteorology, 121, 19 -35.

Jones, H. G. 1992. Plants and microclimate: a quantitative approach to environmental plant physiology. 2 ed. Cambridge University Press.

Kaise, H. F. 1958. The varimax criterion for analytic rotation in factor - analysis. Psychometrika, 23, 187-200.

Kerbauy, G. B. 2004. Fisiologia Vegetal. Rio de Janeiro/Guanabara Koogan S.A.

Krasenshy, J.; Jonak, C. 2012. Drought, salt, and temperature stress-induced metabolic rearrangements and regulatory networks. Journal of Experimental Botany, 63, 1593-1608.

Kriedemann, P. E.; Cromer, R. N. 1996. The nutritional physiology of the Eucalypts - Nutrition and Growth. In: Attiwill, P. M.; Adams, M. A. Nutrition of Eucalypts. Austrália/CSIRIO, pp.109-122.

Kozlowski, T. T.; Kramer, P. J., Pallardy S. G. 1991. The physiological ecology of wood plants. Academic Press.

Laclau, J. P.; Ranger, J. ; Deleporte, P. ; Nouvellon, Y. ; Saint - André, L. ; Marlet, S.; Bouillet, J . P.; 2005. Nutrient cycling in a clonal stand of Eucalyptus and an adjacent savanna ecosystem in Congo: 3. Input-output budgets and consequences for the sustainability of the plantations. Forest Ecology and Management, 210, 375-391.

Laclau, J. P.; Poggiani, F.; Ranger, J.; Gonçalves, J. L. M.; Stape, J. L.; Lima, W. P. de. 2007. Processos de transferência e balanço de água e de nutrientes em povoamentos de Eucalyptus sp. que receberam aplicações de nitrogênio e de biossólidos: reflexos sobre a sustentabilidade. Relatório apresentado a Fundação de Amparo à Pesquisa do Estado de São Paulo - FAPESP.

Larcher, W. 2000. Ecofisiologia vegetal. São Carlos/RiMa.

Leite, F. P., Silva, I. R., Novais, R. F., Barros, N. F., Neves, J. C. L., Villani, E. M. A. 2011. Nutrient relations during an Eucalyptus cycle at different population densities. R. Bras. Ci. Solo, 35, 949959.

Lemos, C. C. Z. Aprimoramentos, teste e uso do modelo 3-PG em plantios clonais de Eucalyptus no nordeste do estado de São Paulo. 2012. In: Ciências Florestais. USP, Piracicaba - SP, pp. 175. 
Luu, T. C.; Binkley, D.; Stape, J. L. 2013. Neighborhood uniformity increases growth of individual Eucalyptus trees. Forest Ecology and Management, 289, 90-97.

Macedo, R. L. G.; Venturini, R. P.; Venturin, N.; Vale, R. S.; Vale, F. A. F. 2014. Plasticidade ecofisiológica. In: Eucaliptocultura no Brasil. Viçosa/UFV, pp. 421- 440.

Melo, E. A. S. C.; Gonçalves, J. L. M.; Rocha, J. H. T.; Hakamada, R. E.; Bazani, J. H.; Wenzel, A. V. A.; Arthur Júnior, J. C.; Borges, J. S.; Malheiros, R. R.; Lemos, C. C. Z.; Ferreira, E. V. O.; Ferraz, A. V. 2016. Responses of Clonal Eucalypt Plantations to N, P and K Fertilizer Application in Different Edaphoclimatic Conditions. Forests, 7, 1-15.

Nikinmaa, E.; Sievänem, R.; Höltta, T. 2014. Dynamics of leaf gas exchange, xylem and phloem transport, water potential and carbohydrate concentration in a realistic 3-D model tree crown. Annals of Botany, 144, 653-666.

Novais, R.F.; Barros, N.F.; Neves, J.C.L. 1986. Interpretação de análise química do solo para o crescimento e desenvolvimento de Eucalyptus spp.: Níveis críticos de implantação e manutenção. Rev. Árvore, 10, 105-111.

Oladi, R., Elzami, E., Pourtahamasi, K., Brauning, A. 2017. Weather factors controlling growth of Oriental beech are on turn over the growing season. Eur J Forest Res., 136, 345-356.

PBMC - Painel Brasileiro de Mudanças Climáticas. 2013: contribuição do Grupo de Trabalho ao primeiro Relatório de Avaliação Nacional do Painel Brasileiro de Mudanças Climáticas: Rio de Janeiro: Sumário Executivo GT1. 24 p

Phillips, N. G.; Ryan, M. G.; Bond, B. J.; McDowell, N. G.; Hinckley, T. M.; Cermak, J. 2003. Tree Physiology, 23, 237-245.

Powers, R. F. 1999. On the sustainable productivity of planted forests. New Forests, 17, 263-306.

Rocha, J. H. T.; Marques, E. R. G.; Gonçalves, J. L. D. M.; Hubner, A.; Brandani, C. B.; Ferraz, A. D. V.; Moreira, R. M. 2016. Decomposition rates of forest residues and soil fertility after clearcutting of stands in response to site managmenet and fertilizer application. Soil Use and Management, 33, 289-302.

Rody, Y. P., Ribeiro, A., Pezzopane, J. E. M., Gleriani, J. M., Almeida, A. Q., Leite, F. P. 2014. Estimativas de índice de área foliar utilizando LAI-2000 e fotografias hemisféricas em plantios de eucalipto. Ciência Florestal, 24, 925-934.

Rolim, G. D. S., Sentelhas, P. C., \& Barbieri, V. 1998. Planilhas no ambiente EXCEL TM para os cálculos de balanços hídricos: normal, seqüencial, de cultura e de produtividade real e potencial. Rev. Brasileira de Agrometeorologia, 6 (1), 133-137.

Ryan, M.G., Stape, J. L., Binkley, D., Fonseca, S., Loos, R., Takahashi, E. N., Silva., C. R.., Silva, S., Hakamada, R. E., Ferreira, J. M. A., Lima, A. M. N., Gava, J. L., Leite, F. P., Andrade, H. B.; Alves, J. M.; Silva, G. G. C. 2010. Factors controlling Eucalyptus productivity: how resource availability and stand structure alter production and carbon allocation. Forest Ecology and Management, 259, 1659-1703. 
Santana, R. C.; Barros, N. F.; Leite, H. G., Comerford, N. B., Novais, R. F. 2008. Estimativa de biomassa de plantações de eucalipto no Brasil. R. Árvore, 32, 697-706.

Santana, R. C., Barros, N. F., Neves, J. C. L. 2002. Eficiência de utilização de nutrientes e sustentabilidade da produção em procedências de Eucalyptus grandis e Eucalyptus saligna em sítios florestais do Estado de São Paulo. R. Árvore, 26, 447-457.

Sette Jr., C. R., Tomazello Filho, M., Lousada, J. L., Lopes, D., Laclau, J. P. 2016. Relationship between climate variables, trunk growth rate and wood density of Eucalyptus grandis W. Mill ex Maiden trees. R. Árvore, 40, 337-346.

Sette JR., C. R.; Tomazello Filho, M.; Lousada, J. L.; Laclau, J. P. 2012. Seasonal increment in trunk diameter of Eucalyptus grandis tress applying dendrometer bands. Ciência Florestal, 22, 763-775

Sette JR, C. R; Tomazello Filho, M.; Dias, C. T. S.; Laclau, J. P. Growth in diameter of Eucalyptus grandis W. Hill. Ex Maiden trees and relationship with climatic variables and mineral fertilization. 2010. Scientia Forestalis, 34, 979-990.

Sevanto, S.; Vesala, T.; Peramaki, M.; Nikinmaa, E. 2002. Time lags for xylem and stem diameter variations in a Scots pine tree. Plant, Cell and Environment, 25, 1071-1077.

Silva, R. M. L.; Hakamada, R. E.; Bazani, J. H.; Otto, M. S. G.; Stape, J. L. 2016. Fertilization response, light use, and growth efficiency in Eucalyptus plantations across soil and climatic gradients in Brazil. Forests, 7, 1-12.

Shimamoto, C. Y.; Botosso, P. C.; Amano, E.; Marques, M. C. M. Stem growth rhythms in trees of a tropical rainforest in Southern Brazil. Trees, 30, 99-111.

Smerthurst, P. J. 2010. Forest fertilization: Trends in knowledge and practice compared to agriculture. Plant and Soil, 335, 83-100.

Snowdon, P. Nutricional disorders and other abiotic stresses of Eucalypts. In: Keane, P. J.; Kile, G. A., Podger, F. D.; Brown, B. N. Diseases and pathogens of Eucalypts. Austrália/CSIRO, pp. 385403.

Soares, R. V.; Batista, A. C.; Tetto, A. F. 2015. Meteorologia e Climatologia Florestal. Curitiba: Os autores.

Souza, M. J. H.; Ribeiro, A.; Leite, H.G., Leite, F. P.; Minuzzi, B. 2006. Disponibilidade hídrica do solo e produtividade do eucalipto em três regiões da Bacia do Rio Doce. Revista Árvore, 30, 399410.

Sfautsch, S.; Renard, J.; Tjoelker, M. G.; Salih, A. 2015a. Phloem as capacitor: radial transfer of water into xylem of tree stems occurs via symplastic transport in ray parenchyma. Plant Physiology, 167, 963-971.

Sfautsch, S.; Teemu, H.; Mencuccini, M. 2015b. Hydraulic functioning of tree stems - fusing ray anatomy, radial transfer and capacitance. Tree Physiology, 35, 706-722.

Sfautsch, S.; Adams, M. A. 2013. Water flux of Eucalyptus regnans: defying summer drought and a record heatwave in 2009. Oecologia., 127, 317-326. 
Stape, J. L.; Binkley, D., Ryan, M. G.; Fonseca, S.; Loos, R. A.; Takahashi, E. N.; Silva, C. R.; Sila, S. R.; Hakamada, R. E.; Ferreira, J. M DE A.; Lima, A. M. N.; Gava, J. L.; Leite, F. P.; Andrade, H. B.; Alves, J. M.; Silva, G. G. C.; Azevedo, M. R. 2010. The Brazil Eucalyptus Potential Productivity Project: Influence of water, nutrients and stand uniformity on wood production. Forest Ecology and Management, 259, 1684-1694.

Stape, J. L., Binkley, D., Jacob, W. S., Takahashi, E. N. 2006. A twin-plot approuch to determine nutrient limitation and potential productivity in Eucalyptus plantations at landscape scales in Brazil. Forest Ecology and Management, 223, 358-362.

Stape, J. L.; Binkley, D.; Ryan, M. G. 2004. Eucalyptus production and the supply, use and efficiency of use of water, light, and nitrogen across a geographic gradient in Brazil. Forest Ecology and Management, 193, 17-31.

Swaif, T., Schepper, V., Vandegehuchte, W. V., Steppe, K. 2015. Stem diameter variations as a versatile research tool in ecophysiology. Tree Physiology, 35, 1047-1061.

Taiz, L.; Zeiger, E.; Moller, I. A.; Murphy, A. 2017. Fisiologia e desenvolvimento vegetal. 6 ed. Porto Alegre/Artmed.

Thornthwaite, C.W.; Mather, J.R. 1955. The water balance. Centerton/Drexel Institute of Technology, Laboratory of Climatology.

Viera, M., Fernandéz, F. R., Rodríguez-Soalleiro, R. 2016. Nutritional prescriptions for Eucalyptus plantations: lessons learned from Spain. Forests, 7, 1-15.

Xavier, A. C.; Soares, J. V.; Almeida, A. C. 2002. Variação do índice de área foliar em clones de eucalipto ao longo de seu ciclo de crescimento. Revista Árvore, 26, 421-427.

Zweifel, R., Drew, D. M., Schweingruber, F., Downes, G. M. 2014. Xylem as the main origin of stem radius changes in Eucalyptus. Functional Plant Biology, 41, 520-534.

Zweifel, R.; Häsler, R. 2001. Link between diurnal stem radius changes and tree water relations. Tree Physiology, 21, $869-877$.

Zweifel, R.; Häsler, R. 2001. Dynamics of water storage in mature subalpine Picea abies: temporal and spatial patterns of chance in stem radius.. Tree Physiology, 21, 561-569.

Zweifel, R.; Item. H.; Häsler, R. 2000. Stem radius changes and their relation to stored water in stems of young Norway spruce tress. Trees. 15, 50-57.

White, D. A., Crombie, D. S., Kinal, J., Bataglia, M., McGrath, J. F., Mendham, D. S., Walker, S. N. 2009. Managing productivity and drought risk in Eucalyptus globulus plantations in south-western Australia. Forest Ecology Management, 259, 33-44.

Wenzel, A. V. A., Gonçalves, J. L. M., Melo, E. A. S. C., Arthur Júnior, J. C., Vomero, P. A. S. Z. 2015. Biomassa e nutrientes de uma plantação de E. urophylla vs. globulus submetida à diferentes níveis de fertilização mineral. In: Estágio Vivencial em Engenharia Florestal. USP. Piracicaba-SP. 
White, D. A., Turner, N. C., Galbraith, J. H. 2000. Leaf water relations and stomatal behavior of four allopatric Eucalyptus species planted in Mediterranean southwestern Australia. Tree Physiology, 20, $1157-1165$.

Whitehead, D; Beadle, C. 2004. Physiological regulation of procivity and water use in Eucalyptus: a review. Forest Ecology and Management, 193, 113-140. 